Journal of Engineering and Applied Sciences 14 (5): 1564-1570, 2019

ISSN: 1816-949X

(C) Medwell Journals, 2019

\title{
Intelligent System for Electromyography (EMG) Signals Classification
}

\author{
Mahmood Khaleel Awsaj and Rabah Nory Farhan \\ Department of Computer Science, University of Anbar, Ramadi, Iraq
}

\begin{abstract}
Muscles in the hands of the human are one of the important parts that depend on the performance of hand dutiest hrough movement which helps the man in the performance of daily functions which carry things and touch and feel everything around him. Muscle signals extracted by EMG system are used to diagnose muscle signals and classify them as normal or abnormal. The process of detecting and classifying EMG signals are difficult and exhausting process and require effort by the specialist doctor to diagnose them. Muscle injury treatment is complicated and require surgical intervention when the injury is severe but when early detection of injury may be treated without surgical intervention. In this study will use the EMG signalsthat have been collected as data. At the beginning extracting, the signals by tracking each signal. These signals are then analyze dusing DWT for muscle bands extraction that is used in feature extraction and using SVM for classification. DWT used for tofeature extraction.
\end{abstract}

Key words: EMG signal, muscular disease, signal processing, DWT, SVM, extraction

\section{INTRODUCTION}

Several years ago, researchers began working on the detection and classification of special signals of human arm muscles and the diagnosis and treatment of diseases and treatment through early detection.

Presented a real-time EMG classifier of user selected intentional movements. EMG signals were recorded from the forearm extensor and flexor muscles of seven able-bodied participants and one congenital amputee. The feature space was segmented using fuzzy C-means clustering. It facilitated the development of dynamic upper extremity prosthesis control strategies using arbitrary, user-preferred muscle contractions (Momen et al., 2007).

Proposed structure neuro classification and neruo-recognition for human arm muscles based amplitude of EMG signals as feature extraction with Artificial Neural Network (ANN). EMG simulator was used to generate an EMG data related to clinical specification and hence, these data were used to simulate an EMG signal using EMGLAB Software. The results showed that, the ability of the proposed structure to classify and recognize the movements of human arm based EMG signals (Al-Faiz and Al-Mashhadany, 2009).

Presented that Root Mean Square (RMS) of the surface EMG signals obtained from Dxtensor Digitorum Superficialis (EDS) muscle during flexion-extension rotation of the index finger at different speeds showed hysteresis. Six different neural network committees were developed to predict the joint angle from the RMS of the SEMG signal. During testing, the neural network committees were able to predict the joint (Shrirao et al., 2009).

Literature review: There are many literatures used different techniques for analysis and classification EMG signals, some of these researches are:

By Goen (2014), the main purpose of the EMG is to analyze and know the electrical activity of the muscles and skeletons during the contraction of the weak and intensive and during the complete rest. The EMG is a complex signal generated from the muscle fibers of $\mathrm{Mo}$, shape, capacity and duration vary in individual muscles, according to a number of factors including the number of muscle fibers of Mo, spatial distribution of end plates and age subject. In addition, individual muscle pulses differ from each other and it is considered in sufficient to evaluate one or a large number of parallelizes and can identify and track synapses using one of the techniques of pattern recognition. The resulting information can be used to determine and determine the causes of the disease, namely neuropathy or myopathy. If a patient maintains a low level of contraction in his muscles, it is possible to identify individual ligaments in an easy way, since, there is a small number of mousse to be active. As the intensity of the contraction increases, a large number of data are recruited, interfering in different sequences, causing the pattern of interference (i.e., the place of imposition), the decomposition of the EMG and the classification of dates in several groups of similar

Corresponding Author: Mahmood Khaleel Awsaj, Department of Computer Science, University of Anbar, Ramadi, Iraq 
forms to give important information for the diagnosis and evaluation of muscular and neurological diseases. Here, recent advances in computer technology have enabled the analysis and evaluation of electromagnetic groups. There are alarge number of quantitative algorithms that are used in the EMG process that are commercially available or advanced. But none of them are widely accepted for widespread clinical use (Goen, 2014).

By Bajaj and Pachori (2012), the researche rpresented a proposal for the Experimental pattern Decomposition (EMD) algorithm that disables the signals of the neural planning in sub-band signals. And it is possible to consider the functions of the intrinsic pattern that resulted from a method as a set of AM-FM signals of amplitude and frequency. The frequency bandwidth and amplitude bandwidth computed from IMFs analytic are used as inputs to Least-Squares Support Vector Machine (LS-SVM) for classification (Bajaj and Pachori (2012).

By Kalaivani et al. (2014), Distributed Wavelet Transform (DWT) is used to analyze EEG waves to sub-band waves and uses feature extraction methods to extract the time range and frequency domain features of the EEG signal used the Genetic algorithm. In this proposed to extract the best features by identifying the relevant traits by removing the features with little or no prophetic information and $\mathrm{k}$-means the workbook to classify the EEG signaling (Kalaivani et al., 2014).

By Kehri et al. (2017), artificial intelligence techniques are used effectively in medical diagnostic tools to increase accuracy in diagnosis and increase knowledge. The importance of Electromyography signals (EMG) in biomedical applications and clinical applications has increased. The detection, treatment and classification of EMG signals is highly desirable because it gives greater evaluation through the unification and distinction between various boneand nerve diseases. Two briefs were reviewed and explained on the different characteristics of the extraction and classification of all techniques used to classify EMG signals. Wavelet Transform (WT), Principle Component Analysis (PCA) as well as Independent Component Analysis (ICA) are different feature extraction techniques. In addition, there are techniques for the classification of data (EMG) such as Probabilistic Neural Network (PNN), Support Vector Machine (SVM), Artificial Neural Networks (ANN), etc. Here, muscle and neurological diseases are categorized through muscle planning signals (EMG) through different combinations of methods of feature extraction and types of workbooks. The EMG signal is a highly complex and non-linear signal that is widely used for the clinical diagnosis of neurological problems and neuromuscular problems. This complication causes researchers and experienced individuals to fail to provide adequate information about these signals. Where, these signals are noisy by transferring the voltage difference between the electrodes to the computer arrives to display the voltage tracking of the potential recordings. As there are many neurological and muscular disorders that affect the spinal cord that any discovery and treatment early helps the human to getrid of complications. And the elimination of this noise is done through the use of Fast Fourier Transform (FFT) for the analysis of signals (EMG) (Kehri et al., 2017).

By Riaz et al. (2016), the researcher provided a method for classifying the waves of the neuro-nervous planning according to Empirical Mode Decomposition (EMD) for the purpose of extracting the features. The feature extracted by EMD fed into a Support Vector Machine (SVM) which is used for classification. It uses a kernel to convert the input signal toa higher dimensional space. In this way, we provide samples of the classification capable of optimization and therefore, used in a wide range of applications of pattern recognition where it is done using the proposed accuracy of the general database which deals with all problems and different classification including identification and detection of diseases and data suffered by patients (Riaz et al., 2016).

\section{MATERIALS AND METHODS}

This research contains involves preprocessing, extracting the features and classification, Fig. 1 shows block diagram of the researches.

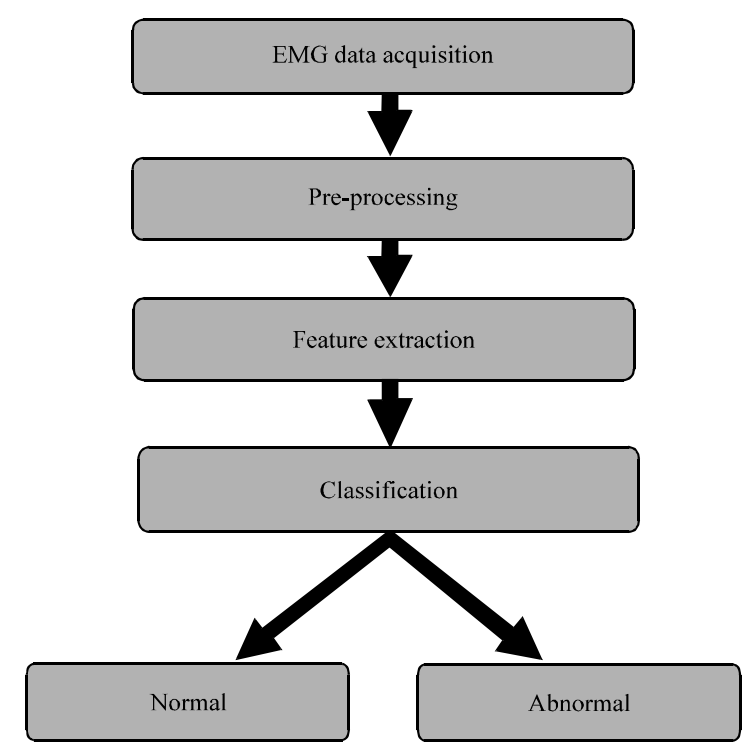

Fig. 1: System block diagram 
EMG data acquisition: The EMG data were collected from http://www.emglab.netfor which was recorded by the researcher M. Nikolic which he collected from many people and both sexes. The samples were collected from collection group patients, healthy and unhealthy. Each state contains of many signals. The data recorded with duration of 50's.

The data consists of two groups. The first group is for healthy people aged between (21-37) years and of both sexes. There were 10 people. All these people do not suffer from any muscle or neurological disorder. The second group was infected with neurological disorders, aged between (19-63) years and of both sexes with a total of 7 people, all of whom were suffering from neurological illness. The brachial biceps and medial vastus muscles were chosen for study asthe most common muscle in all cases.

The EMG signals were recorded under the usual conditions for MUAP analysis:

- The recordings were made at low (just above threshold) voluntary and constant level of contraction

- Visual and audio feedback was used to monitor the signal quality

- A standard concentric needle electrode was used

- The EMG signals were recorded from 5 places in the muscle at three levels of insertion (deep, medium, low)

- The high and low pass filters of the EMG amplifier were set at $2 \mathrm{~Hz}$ and $10 \mathrm{kHz}$

Pre-processing: EMG waves are very noisy and mixed with massive quantity of useless data generated by physiological artifacts that cover the EMG waves, filtering the waves are useful to eliminate this noise and remove unwanted data to obtain filtered signal (Tolic and Jovic, 2013). So, all these waves pass to pre-processing to remove noises caused by eye blinking, deep breathing, muscle movements, etc. The high frequency removed and filtered by Low Pass Filter (LPF), so, low frequencies will pass that hold the original EMG signal that contain features and important data about muscle activities. The data pre-processing aim is to enhance the levels of signals of attention whereas attenuating or dismissing undesirable signals in noisy recordings of the signal (Borhade and Nagmode, 2016). Signal processing filters are more helpful while it comes to repressing the high frequencies in the signal, smoothing the signal, decreasing the noise or repressing the low frequencies (Seitz, 2010) (Fig. 2). (a)

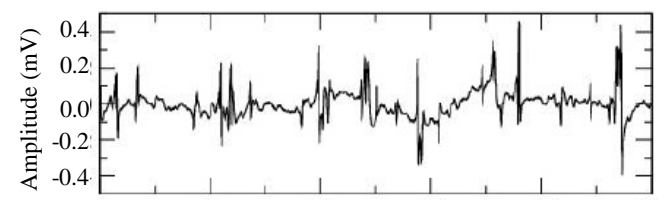

(b)

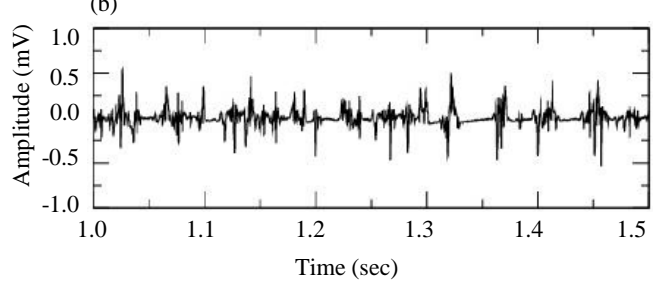

Fig. 2: a) Normal EMG signal and b) Abnormal EMG signal

Muscle signals are subjected to noise and non-useful data that conceal the signal properties that affect the data classification process. During the signal recording process, the signal is combined with the noise caused by a number of factors including muscle movement, signal transmission modes and other factors that interfere with the signal. The purpose of the pre-treatment is to eliminate this noise and extract the original signal from all these noises and to enhance levels of attention signals and remove unwanted signals. So, all these waves go to pretreatment to remove noise. The original signal falls within 20-1000 frequencies and the noise produces frequencies $>1000 \mathrm{~Hz}$. Therefore, all frequencies are considered $>1000 \mathrm{~Hz}$ noise. The high frequency is removed and filtered by a band pass filter, so, lower frequency will pass, holding the original EMG signal containing important features and data about muscle activities.

The filter used in the proposed system for high frequency, removing infinite Impulse Response (FIR). FIR filter designed to pass frequency $<1000 \mathrm{~Hz}$. Algorithm 1 shows preprocessing steps:

\author{
Algorithm 1; Pre-processing stage: \\ Input: signal \\ $\mathrm{N}:$ Number of orders \\ Normalize filter: $\mathrm{FS}=2 \mathrm{kH}$ \\ Low fc: $20 \mathrm{~h}$ \\ High fc: $1 \mathrm{kHz}$ \\ Output: filtered signals \\ 1. Load sign from EMGLAB \\ 2. Normalize frequency \\ $\mathrm{nf}=(2 / \mathrm{Fs}) * \mathrm{fc}$ \\ 3. FIR filter formation \\ FIR = Firl(n, nf, 'bandpass') \\ 4. Filter the signal \\ Signal $=$ filter $(F I R, 1$, signal $)$ \\ 5. Return signal
}

Feature extraction: The method for extraction the important features from the signal that contain information about signal to reduce the dimensionality size of the 
signal. The requirement of feature extraction method is to extract right features or verifying what's appropriate data that is used to classify EMG signal (Punskaya, 2009). The basic characteristics of the signal are immersed in the noise. Therefore, in order to extract the features, the muscle signals are analyzed in order to give an accurate description of the energy of the signal as a function of time and frequency domain (Fatehi and Suleiman, 2011). Extract the features from EMG signals are searching for characteristics of signal that describe the activities of muscle. Extract the features also minimizes the data quantity that is used in classification. Finding features that appropriate are often decisive for effective classification (Tolic and Jovic, 2013). The way for important features extracted from the signal which contain the characteristics of the signal, this process reduces the dimensional size of the signal. In this study, we derive the special features of the signal which distinguish each signal from other signals. The process of extraction of features is very important for very good classification. In this proposed, we will extract here only the low band of DWT outputs were taken for features extraction. The Haar DWT method is used toextract the features. DWT is one of the best and most efficient methods for analyzing the signal and extracting the muscle signal.

After extracting the low band, we will explain each feature parameters that have been used to extract the signal.

Integrated EMG: Integrated EMG (IEMG) is calculated as the summation of the absolute values of the EMG signal amplitude. Generally, IEMG is used as an onset index to detect the muscle activity that used to on coming the control command of the assistive control device. It is related to the $\mathrm{EMG}$ signal sequence firing point:

$$
\operatorname{IEMG}=\sum_{\mathrm{n}=1}^{\mathrm{N}}\left|\mathrm{x}_{\mathrm{n}}\right|
$$

Where:

$\mathrm{N}=$ The length of the signal

$\mathrm{x}_{\mathrm{n}}=$ The EMG signal in a segment

Mean absolute value: Mean Absolute Value (MAV) is similar to Average Rectified Value (ARV). It can be calculated using the moving average of full-wave rectified EMG. In other words, it is calculated by taking the average of the absolute value of the EMG signal. It is an easy way for detection of muscle contraction levels and it is a popular feature used in the myoelectric control application. It is defined as:

$$
\mathrm{MAV}=\frac{1}{\mathrm{~N}} \sum_{\mathrm{n}=1}^{\mathrm{N}}\left|\mathrm{x}_{\mathrm{n}}\right|
$$

Modified mean absolute value: Modified Mean Absolute Value (MMAV) is an extension of MAV using weighting window functions $\mathrm{w}_{\mathrm{x}}$. It is shown as:

$$
\begin{aligned}
& \text { MMAV }=\frac{1}{\mathrm{~N}} \sum_{\mathrm{n}=1}^{\mathrm{N}} \mathrm{w}_{\mathrm{z}}\left|\mathrm{x}_{\mathrm{n}}\right| \\
& \mathrm{w}_{\mathrm{x}}= \begin{cases}1 \text { if } 0.25 \mathrm{~N} \leq \mathrm{n} \leq 0.75 \mathrm{~N} \\
0.5 & \text { otherwise }\end{cases}
\end{aligned}
$$

Variance of EMG: Variance of EMG (VAR) uses the power of the EMG signal as a feature. Generally, the variance is the mean value of the square of the deviation of that variable. However, the mean of EMG signal is close to zero. In consequence, variance of EMG can be calculated by:

$$
\operatorname{VAR}=\frac{1}{\mathrm{~N}-1} \sum_{\mathrm{n}=1}^{\mathrm{N}} \mathrm{x}_{\mathrm{n}}^{2}
$$

Waveform length: Waveform Length (WL) is the cumulative length of the waveform over the time segment. WL is related to the waveform amplitude, frequency and time. It is given by Algorithm 2:

$$
\mathrm{WL}=\sum_{\mathrm{n}=1}^{\mathrm{N}-1}\left|\mathrm{x}_{\mathrm{n}+1}-\mathrm{X}_{\mathrm{n}}\right|
$$

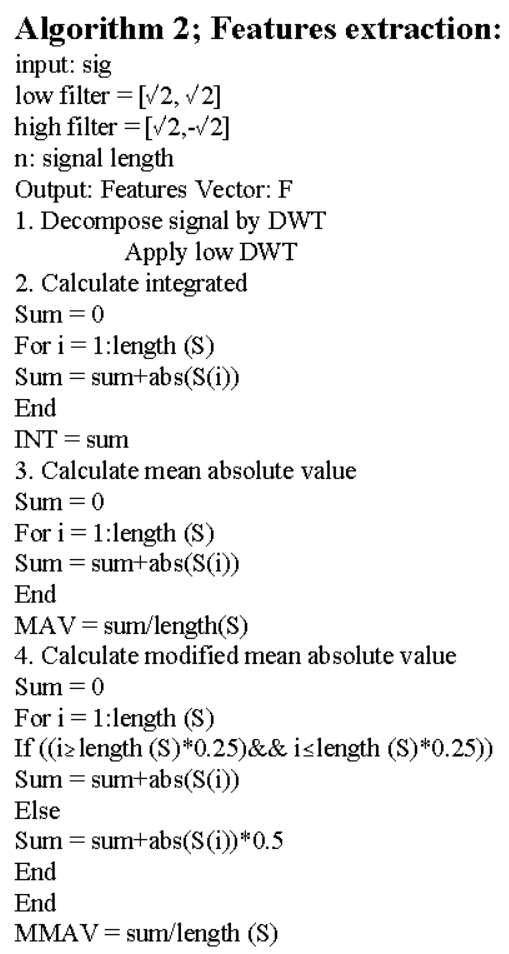




\section{J. Eng. Applied Sci., 14 (5): 1564-1570, 2019}

5. Calculate variance

Sum $=0$

For $\mathrm{i}=1$ : length $(\mathrm{S})$

Sum $=\operatorname{sum}+(\mathrm{S}(\mathrm{i}))^{2}$

End

$\mathrm{VAR}=$ sum/length(S)-1

6. Calculate waveform length

Sum $=0$

For $\mathrm{i}=1$ : length $(\mathrm{S})-1$

Sum $=\operatorname{sum}+\operatorname{abs}(S(i+1))-S(i)$

End

$\mathrm{WL}=$ sum

7. $\mathrm{F}=[\mathrm{INT}, \mathrm{MAV}, \mathrm{MMAV}, \mathrm{VAR}, \mathrm{WL}]$

8. Return $\mathrm{F}$

Classification: At this stage the signals are classified as normal and abnormal by the SVM. To classify the signals, the feature vector extracted from the previous phase is used and feed into the SVM to be classified.

Support vector machine: The classifiers are now applied using the features computed in the previous sub-section that includes discrete wavelet transform of the EMG signals and features like IEMG, MAV, MMAV, VAR and wave form length for the D4 coefficient of the transform. These features are now utilized to train the SVM Model and then test on the same trained SVM for the classification. SVM (Support Vector Machine) is a classifier which requires training of the model for the testing of the samples and such technique in which training is given is called as supervised learning technique. In this, the model is firstlytrained to learn according to the features of the classes which need to be classified. Then that trained model is exploited in the classification process. Learning given to the classifier provides better results compared to the other classifiers in which unsupervised learning is used. This classifier is simple and easy to understand as it constructs a hyperplane between the different classes which need to be classified. The classifier used may be linear or nonlinear. In linear SVM, training samples of the classes are linearly separable. But itis very difficult in practice situations that a straight line is sufficient to classify each and every sample. For such cases, non-linear classifier is exploited (Algorithm 3).

\footnotetext{
Algorithm 3; SVM classification:

Input: feature vector

Parameters:

Input vector: $\mathrm{x}\{\mathrm{i}\}$

Load training sample: $y$

Output: classification result

1. Train SVM on training sample y

svmStruct $=$ svmtrain $(y)$
}

\author{
2. Classify $x$ using SVM \\ for each $\mathrm{x}$ input apply SVM classifier functionc

$$
c=\sum_{i} \propto_{i}\left(s_{i}, x\right)+b
$$ \\ 3. Retum classification result
}

\section{RESULTS AND DISCUSSION}

The system was implemented on data consisting of 185 normal signals and 100 abnormal signals to be the total data 285 signals. These data were divided into two groups 100 signals were used for training and 185 others were used for testing. The features used in this study are able to distinguish the differences between signals that leads to a good classification of signals (Table 1). Table 2 and 3 see features from two states. The support vector machine used in the proposed system uses these features to train the system and store it in a database. The proposed system is able to recognize abnormalities in the muscle with accuracy of $96.75 \%$ (Table 4 ). The proposed method DWT has been a practical and useful method for analyzing muscle signals and extract the muscle rhythms and then extract the features. Five features were extracted from each rhythm. These features were trained and categorized by SVM (Fig. 3).

Table 1: Features of five signals for healthy persons

\begin{tabular}{llllll}
\hline No. & INT & MAV & MMAV & VAR & WL \\
\hline Signal 1 & $2.3730 \mathrm{e}+06$ & 18.1056 & 9.0528 & 801.4343 & $1.8334 \mathrm{e}+06$ \\
Signal 2 & $2.8881 \mathrm{e}+06$ & 22.0357 & 11.0178 & $2.3148 \mathrm{e}+03$ & $2.3419 \mathrm{e}+06$ \\
Signal 3 & $1.0898 \mathrm{e}+07$ & 83.1490 & 41.5745 & $1.4473 \mathrm{e}+05$ & $1.9630 \mathrm{e}+07$ \\
Signal 4 & $3.1455 \mathrm{e}+06$ & 23.9995 & 11.9998 & $2.0168 \mathrm{e}+03$ & $2.2187 \mathrm{e}+06$ \\
Signal 5 & $5.9856 \mathrm{e}+06$ & 45.6681 & 22.8341 & $4.1519 \mathrm{e}+03$ & $4.9371 \mathrm{e}+06$ \\
\hline
\end{tabular}

Table 2: Features of five signals for unhealthy persons

\begin{tabular}{lccllc}
\hline No. & INT & MAV & MMAV & VAR & WL \\
\hline Signal 1 & $3.0693 \mathrm{e}+06$ & 23.4179 & 11.7090 & $1.8191 \mathrm{e}+03$ & $2.4123 \mathrm{e}+06$ \\
Signal 2 & $7.8956 \mathrm{e}+06$ & 60.2407 & 30.1203 & $8.7059 \mathrm{e}+03$ & $7.1485 \mathrm{e}+06$ \\
Signal 3 & $2.4226 \mathrm{e}+06$ & 18.4839 & 9.2420 & $1.0997 \mathrm{e}+03$ & $1.7043 \mathrm{e}+06$ \\
Signal 4 & $5.2507 \mathrm{e}+06$ & 40.0613 & 20.0307 & $4.8292 \mathrm{e}+03$ & $4.5253 \mathrm{e}+06$ \\
Signal 5 & $2.2537 \mathrm{e}+06$ & 17.1950 & 8.5975 & 904.4113 & $1.4514 \mathrm{e}+06$ \\
\hline
\end{tabular}

Table 3: Features from normal data

\begin{tabular}{lclllc}
\hline No. & Feature 1 & Feature 2 & Feature 3 & Feature 4 & Feature 5 \\
\hline Signal 1 & $5.5735 \mathrm{e}+06$ & 42.5238 & 21.2619 & $3.3683 \mathrm{e}+03$ & $5.5334 \mathrm{e}+06$ \\
Signal 2 & $7.9539 \mathrm{e}+06$ & 60.6859 & 30.3429 & $1.5060 \mathrm{e}+04$ & $4.7816 \mathrm{e}+06$ \\
Signal 3 & $9.2760 \mathrm{e}+05$ & 7.0773 & 3.5386 & 151.1395 & $4.2565 \mathrm{e}+05$ \\
Signal 4 & $9.0162 \mathrm{e}+06$ & 68.7904 & 34.3952 & $1.5760 \mathrm{e}+04$ & $5.9574 \mathrm{e}+06$ \\
Signal 5 & $4.8070 \mathrm{e}+06$ & 36.6760 & 18.3380 & $2.4072 \mathrm{e}+03$ & $8.8856 \mathrm{e}+06$ \\
\hline
\end{tabular}

Table 4: Features from abnormal data

\begin{tabular}{lcllcc}
\hline No. & Feature 1 & Feature 2 & Feature 3 & Feature 4 & Feature 5 \\
\hline Signal 1 & $1.8629 \mathrm{e}+06$ & 14.2135 & 7.1067 & $1.0511 \mathrm{e}+03$ & $1.8030 \mathrm{e}+06$ \\
Signal 2 & $2.4497 \mathrm{e}+07$ & 186.9016 & 93.4508 & $2.5710 \mathrm{e}+05$ & $3.0035 \mathrm{e}+07$ \\
Signal 3 & $5.9304 \mathrm{e}+06$ & 45.2473 & 22.6236 & $6.1122 \mathrm{e}+03$ & $6.7300 \mathrm{e}+06$ \\
Signal 4 & $2.7211 \mathrm{e}+06$ & 20.7613 & 10.3807 & $1.4351 \mathrm{e}+03$ & $2.2352 \mathrm{e}+06$ \\
Signal 5 & $2.7284 \mathrm{e}+06$ & 20.8166 & 10.4083 & $1.1159 \mathrm{e}+03$ & $2.3807 \mathrm{e}+06$ \\
\hline
\end{tabular}




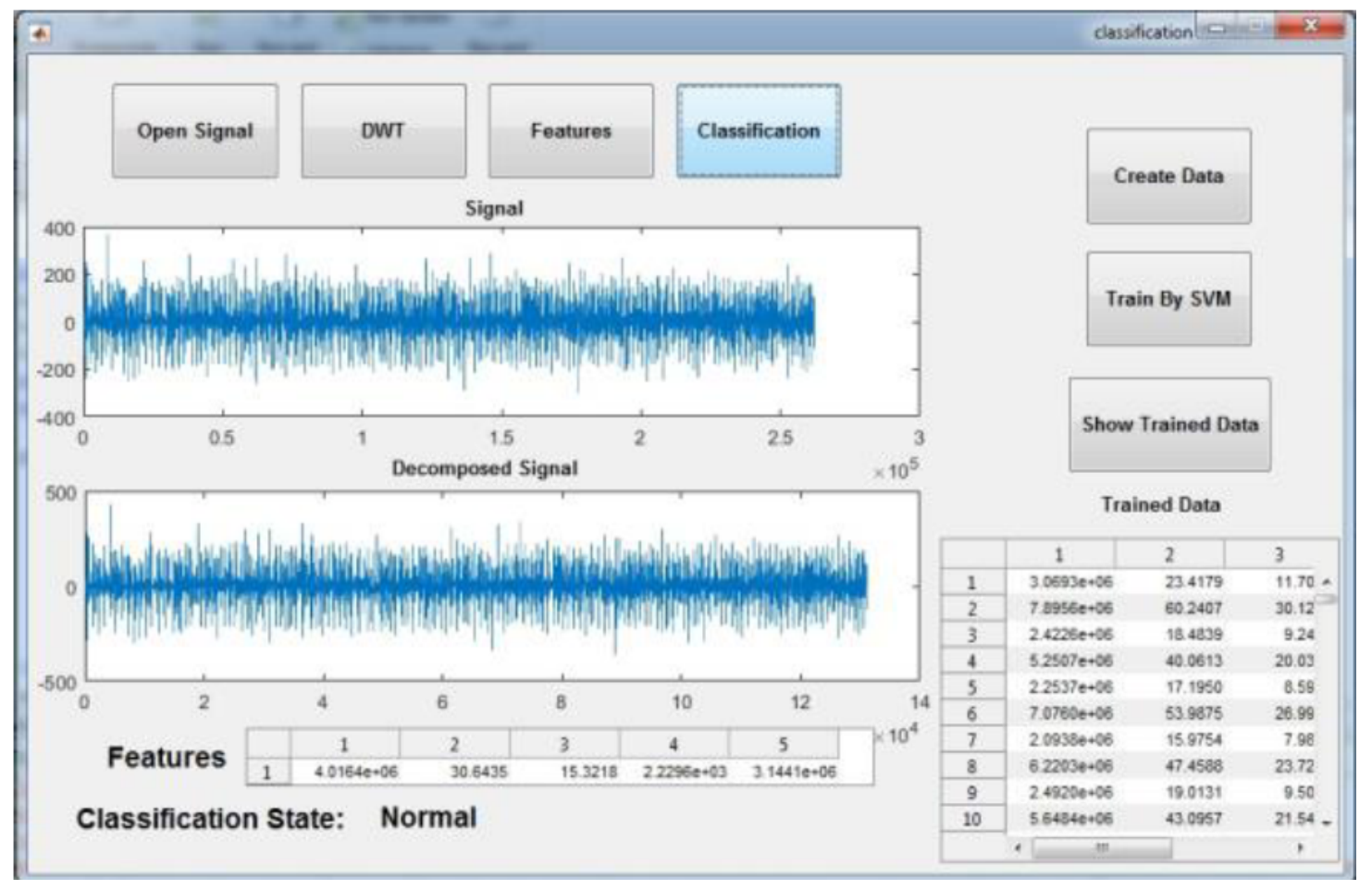

Fig. 3: SVM classification of the signals

\section{CONCLUSION}

The most common way to record muscle activities is the EMG system. EMG signals are complex signals that contain much important information about muscle functions and muscle disorders. Where the EMG has an important role in the detection of muscular dystrophy and inflammation of the nerves and tumors suffered by the muscles. Diagnosis of muscle signal by doctors specializing in musculoskeletal diseases is very difficult as it requires specialized technicians. This takes a long period of time in addition to the financial cost as the early detection of disorders and muscle diseases reduces time, effort and financial expenditure. The system is able to distinguish and classify muscle signals with high accuracy. DWT is applied for signal analysis and extraction. The proposed features give high precision and efficiency in distinguishing between different positions and detecting muscle dysfunction. The use of DWT with SVM in medical applications gives good results and high accuracy. Where the proposed system achieved a good accuracy of $96.75 \%$

\section{REFERENCES}

Al-Faiz, M.Z. and Y.I. Al-Mashhadany, 2009. Human arm movements recognition based on EMG signal. Masaum. J. Basic Appl. Sci., 1: 164-171.
Bajaj, V. and R.B. Pachori, 2012. Classification of seizure and nonseizure EEG signals using empirical mode decomposition. IEEE. Trans. Inf. Technol. Biomed., 16: 1135-1142.

Borhade, R.R. and M.S. Nagmode, 2016. EEG signal classification for detection of epileptic seizures by extracting various features. Proceedings of the 3rd International Conference on Electrical, Electronics, Engineering Trends, Communication, Optimization and Sciences (EEECOS), June 1-2, 2016, Sasi Institute of Technology \& Engineering, Tadepalligudem, Andhra Pradesh, India, pp: 945-948.

Fatehi, T.A.H. and A.B.R. Suleiman, 2011. Features extraction techniques of EEG signals for BCI application. Proceedings of the Arab 12th International Conference on Information Technology, December 11-14, 2011, Naif Arab University for Security Sciences, Riyadh, Saudi Arabia, pp: $35-40$.

Goen, A., 2014. Classification of EMG signals for assessment of neuromuscular disorders. Intl. J. Electron. Electr. Eng., 2: 242-248.

Kalaivani, M., V. Kalaivani and V.A. Devi, 2014. Analysis of EEG signal for the detection of brain abnormalities. Intl. J. Comput. Appl., 2014: 1-6. 
Kehri, V., R. Ingle, R. Awale and S. Oimbe, 2017. Techniques of EMG Signal Analysis and Classification of Neuromuscular Diseases. In: Advances in Intelligent Systems Research, Lyer, B., S. Nalbalwar and R. Pawade (Eds.). Atlantis Press, Milton Park, Didcot, USA., pp: 485-491.

Momen, K., S. Krishnan and T. Chau, 2007. Real-time classification of forearm electromyographic signals corresponding to user-selected intentional movements for multifunction prosthesis control. IEEE. Trans. Neural Syst. Rehabil. Eng., 15: 535-542.

Punskaya, E., 2009. Design of fir filters. MSc Thesis, Columbia University, New York, USA.
Riaz, F., A. Hassan, S. Rehman, I.K. Niazi and K. Dremstrup, 2016. EMD-based temporal and spectral features for the classification of EEG signals using supervised learning. IEEE. Trans. Neural Syst. Rehabil. Eng., 24: 28-35.

Seitz, A., 2010. Basic image processing. Master Thesis, Faculty of Life Sciences, Ecole Polytechnique Federale de Lausanne, Lausanne, Switzerland.

Shrirao, N.A., N.P. Reddy and D.R. Kosuri, 2009. Neural network committees for finger joint angle estimation from surface EMG signals. Biomed. Eng. Online, 8: 1-11.

Tolic, M. and F. Jovic, 2013. Classification of wavelet transformed EEG signals with neural network for imagined mental and motor tasks. Kinesiology Intl. J. Fundam. Appl. Kinesiology, 45: 130-138. 